\title{
Consideration of Service Time in Placing Clients of Web-Based Services
}

\author{
Wei Zhang \\ Worcester Polytechnic Institute \\ weizhang@cs.wpi.edu
}

\author{
Craig E. Wills \\ Worcester Polytechnic Institute \\ cew@cs.wpi.edu
}

\begin{abstract}
Web-based services involving dynamic computation of the content are increasingly used on the Web. This computation may not only involve processing, but often involves access to other back-end services typically for database access. These multitiered Web-based services have different characteristics than traditional Web content and new models need to be considered not only how to manage them, but also how the DNS mechanism should map clients to the appropriate front-end server.

In this work we study the potential of considering back-end service times in the decision of mapping clients to servers. We do so by gathering data from deployed platforms in the Internet today from client locations both scattered around the globe and around the United States. We use these data for the simulation of straightforward policies that account for back-end service time. Our results show that in the best case our simple policies have better performance than using current DNS decisions, while in the worst case they provide comparable performance for a range of performance metrics.
\end{abstract}

\section{INTRODUCTION}

Web-based services involving dynamic computation of the content are increasingly used on the Web. This computation may not only involve processing, but often involves access to other back-end services typically for database access. These services may be in the same data center as the client-facing front-end server or may be located in a remote location. These multi-tiered Web-based services have different characteristics than traditional Web content and new models need to be considered not only how to manage them, but also how to map clients to the appropriate front-end server.

The traditional approach for mapping clients to one of multiple server locations for content is to simply map a client to a nearby data center when that client makes a DNS request for the given server. This is the core of the approach used by content distribution networks and has worked well for static content where the retrieval costs are largely dependent on transporting data over a TCP connection. Shorter round-trip times result in quicker ACKs and shorter response times.

However, Web-based services introduce a potentially significant component that we conjecture needs to be included in the decision of which front-end server to use for a client request. If a nearby front-end server incurs significant backend costs to perform a service then the best front-end server for a client of that service may not be the closest and could even be one much further away. The client-to-server mapping decision made by DNS should not only consider the client and server location, but also account for the nature of the service itself. This consideration is not possible if a Web site offers all services under the same server name, such as WWw. company.com, but increasingly we see sites "expose" Web services, such as search.company. com allowing the desired service to be considered as part of the DNS decision.

In this work we study the potential of considering back-end service times in the decision of mapping clients to servers. We do so by gathering data from deployed platforms in the Internet today from client locations both scattered around the globe and around the United States. We use these data for the simulation of straightforward policies that account for back-end service time. Our results show that in the best case our simple policies have better performance than using current DNS decisions, while in the worst case they provide comparable performance for a range of performance metrics.

In the remainder of this paper we further motivate the problem and our approach in Section II. We describe the study we performed in Section III with the results obtained from a set of clients in the United States in Section IV. We define a simple parameterized set of policies for consideration of service time in Section V and use our gathered data to evaluate the performance of these policies for the U.S. clients and a set of global clients in Section VI. We describe related work in Section VII and conclude with a summary and future work in Section VIII.

\section{Motivation}

As the online social networking, video content hosting, and other Web services become increasingly popular, these services are more complex as they have more back-end dependencies on other services such as database queries. When service providers deploy their services, these dependent services may not be at the same location as the front-end client-facing servers. Hence, fulfillment of a Web service may require remote database queries to be performed. In addition, the resources granted to each service may not be balanced and overloaded back-end servers may have an impact on the performance of front-end application servers. As a result, different front-end application servers may have different service times due to the configuration and current load of their dependent set of back-end servers.

This observation motivates our work to examine the backend processing costs of current Web services and seek to understand if these costs can and should be considered in the 
mapping of clients to front-end servers for these services. If the processing cost of the back-end server is significant then it should be considered when making a decision to choose the best front-end server for a client.

While consideration of back-end processing costs may be worthwhile, an important question is how to study this issue without direct access to deployed Web-service platforms. Rather than seek such access our approach is to use a simple observation about a typical HTTP network flow as shown in Figure 1 to estimate the back-end service time for any transaction.

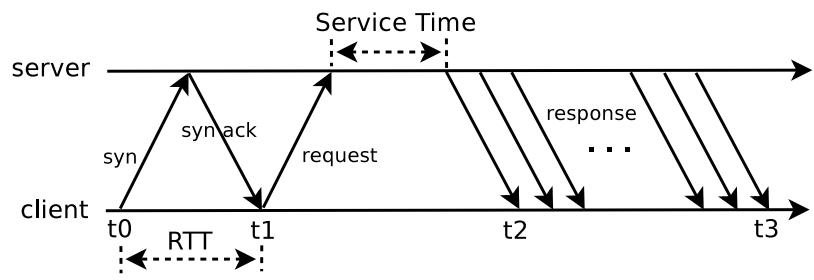

Fig. 1. Network Flow of Typical HTTP Transaction

As shown in the figure, $t_{1}-t_{0}$ is the time to set up the TCP connection and is a typical estimate of the round-trip time (RTT) between client and server. An immediate HTTP GET request is then sent from the client at $t_{1}$ with $t_{2}$ the time in which the first byte of the HTTP response is returned to the client. Data continues to flow to the client (corresponding TCP ACKs from client to server are not shown) until $t_{3}$ when the last byte of the response is received. The time $t_{2}-t_{1}$ contains both a RTT and the time taken at the server before it generates any data to return to the client. The server could begin sending data (such as HTTP response headers) before all servicing is done, but in preliminary testing we found this situation does not occur frequently and when observed the HTTP data arrives within $10 \mathrm{~ms}$ of the HTTP headers so we did not try to distinguish these cases in subsequent testing. Rather, we consider $\left(t_{2}-t_{1}\right)-R T T$ as an estimate of the service processing time. That is, $T_{r t t}=t_{1}-t_{0}$ and $T_{\text {service }}=\left(t_{2}-t_{1}\right)-T_{r t t}$. While this approach does not allow us to distinguish why there is a service processing delay, it does allow us to estimate its magnitude. The delay could be caused by some combination of front-end service delays, back-end service delays or access to remote back-end services. Obviously it is possible that if the subsequent HTTP GET request is delayed in the network then the estimate value of $T_{\text {service }}$ will incorrectly assume the delay is attributed to service time rather than network delay. However if we make multiple requests for a Web service and the requests consistently yield significant estimated service times then we can safely assume that the delays are indeed caused by servicing.

\section{STUDY}

To study this problem we first identified a set of popular Web sites providing a range of services as candidates for testing from a couple of platforms of clients. We then performed an initial set of tests to narrow this candidate set to a smaller number. Once this set is determined we describe the methodology used in studying the services provided by these sites and how we analyzed the collected data.

\section{A. Web-Based Services for Study}

In selecting the set of services to study, we wanted to focus on a relatively small set of popular Web sites that supported for more than one service for study. Using Alexa [1], we choose 20 popular Web sites as candidate service providers to study. For each site, we choose four types of services to represent the whole site, namely, Home Page, Image, Search, and Video Page. The Home Page service is the front page of a Web site, which typically has dynamic content and is expected to have some amount of service time. A relatively small image (typically on the home page) for each site is selected as the Image service. While we expect these static images to be cached on the servers and require minimal service time, we include this "service" to verify the expected results. A Search service is generally involved with sending queries that likely require contacting a back-end server, particularly if the query is not cached by a front-end server. Given that the back-end server could be accessed remotely this service may involve a large service time. The last service we include is for a Video Page. While this service is not directly serving video content its content is dynamic in generating the current set of popular videos.

Given this set of candidate services, we wanted to focus our study on a smaller set of services with two properties. First, we wanted services for which the service time is non-trivial and exhibits some amount of variance indicating that the service time may be a consideration in the overall performance for that service. Second, we wanted services where the size of the "platform", measured in terms of the number of unique IP addresses offering this service, provided multiple opportunities for selection by the DNS mechanism of the service.

To determine a set of services with these properties we identified a URL to retrieve for each of our candidate services. For services involving a search string we made sure to use a different set of search terms on each retrieval. We then performed an initial measurement study from each of the clients in our global set. For the first property, we calculated the service time $T_{\text {service }}$ for each of the services in our candidate set for each retrieval from all 10 global clients and found out most of the services have negligible service timesmany of these are the Image service. However, over $10 \%$ of them have median service times over 200 milliseconds, and several of them have medians over half second. In addition, many services with larger median service time performance also exhibit much variation.

For the second property, we used these preliminary results to collect the list of all IP addresses returned to all ten clients. In some cases multiple IP addresses were returned for a single DNS lookup. In some cases the same IP address(es) were returned to different clients. We did not try to distinguish if multiple IP addresses were associated with the same data center or represented multiple data centers. We merged all IP 
addresses for all clients of services for each of the 20 sites in our candidate set to determine the "platform" of addresses for each site. We found the size of the platform for the $20 \mathrm{Web}$ sites ranged from 1 to 200 IP addresses for a single site.

In considering the two desired properties for selecting services of sites to study we focused on sites with a larger number of IP addresses and with larger and more variable service times. Based on this consideration we selected the four services of the four sites foxnews.com, msn.com, yahoo.com and cnbc.com for focused study.

\section{B. Client Sets}

We used two sets of clients to study the performance of Web-based services on two different scales. In all cases we selected clients for their geographic location from the set of PlanetLab nodes available to use. When multiple PlanetLab nodes were available in a geographic area we selected specific nodes based upon low load and the fewest number of active slices.

The global client set consists of 10 PlanetLab nodes located in different continents of the world. Two of them are in USA (USW(est) and USE(ast)), three of them are in Europe (UK, FR and DE), another two are in Asia (KR and JP) and the others are located in Africa (EG), Australia (AU), and South America (BR) respectively. We choose this set to represent a geographic distribution around the world.

The U.S. clients set consists of 8 PlanetLab nodes geographically dispersed around the United States. These clients are located in the states WA, CA, CO, TX, IL, FL, MD and MA. We choose this set to understand performance variation of services in a particular region of the world in which clients for many of these popular sites are concentrated.

\section{Methodology}

Before looking to measure Web-based service performance, it is important to consider what is meant by performance. The traditional measure of performance for a Web object is the time from when a user requests an object until that object is received in its entirety. Referencing Figure 1 this is the value of $t_{3}-t_{0}$, which includes TCP connection setup as well as the time to make the HTTP request and receive all of the data. However modern browsers begin to process data as they are received for purposes of executing embedded JavaScript, retrieving embedded objects and rendering the contents. This "as data are available" approach argues that it also important to consider how long it takes for the first byte of data to arrive $\left(t_{2}-t_{0}\right)$. These two basic metrics indicate when all data from the service are received and when the client has data to begin work.

Given the typical settings of most browsers to support persistent TCP connections, we can also extend each basic metric to consider persistent connections. With persistent connections, the client does not have to re-establish the connection resulting in more efficiency. This extension leads to four possible performance metrics to consider: $T_{\text {total }}=$ $t_{3}-t_{0}, T_{\text {total-pers }}=t_{3}-t_{1}, T_{\text {firstbyte }}=t_{2}-t_{0}$ and
$T_{\text {firstbyte-pers }}=t_{2}-t_{1}$. In our work, we compute all four metrics, but focus primarily on $T_{\text {firstbyte }}$ as that represents the time that a client can begin processing data and is a metric that is independent of the amount of data returned by a service. We also show some results for $T_{\text {total }}$ as appropriate. While service time is an even bigger contributing component of requests made over persistent connections we assume that Web-based service requests will primarily be made as the first request to a front-end application and thus will likely incur the cost to create a TCP connection. We also focus only on the time for the invocation of the Web-based service and not on any subsequent retrieval of embedded objects as those are often done in parallel with retrieval and parsing of the base content.

While our work evaluates the quality of the DNS decision we do not explicitly account for the cost of this lookup in the work. In a small number (1\%) of cases in our study we also observe the use of HTTP redirection to direct a client to an alternate server. Whenever a redirection occurs, we measure the time for the redirected server so that $t_{0}$ is reset to the time the client initializes a connection to this server.

In analyzing the gathered data we distinguish the results from three particular server IP addresses within each group of results for a service. These three server IP addresses are $S_{d n s}, S_{\text {best-rtt }}$, and $S_{\text {best-service. The server identified by }}$ $S_{d n s}$ is the address chosen by the DNS mechanism during this group of requests. It represents the case that the client always uses the DNS-returned server-the expected behavior for an actual client of this service from the given client location. The server $S_{\text {best-rtt }}$ is the address of the server machine with the lowest $T_{r t t}$ for a group of requests and represents the closest server. While this server cannot be determined ahead of time, it represents a benchmark for the importance of RTT when considering the best performing server. Similarly, the value of $S_{\text {best-service }}$ is the address of the server machine with the lowest $T_{\text {service }}$ for a group of requests and represents the fastest server once it receives the request. Again this value cannot be determined ahead of time, but it represents a benchmark for the importance of service time when considering the best performing server.

The goal in comparing the performance of the server returned by the DNS mechanism $S_{d n s}$ with the server providing the best RTT $S_{\text {best-rtt }}$ and the server providing the best service time $S_{\text {best-service }}$ is to understand how well the current DNS mechanism is doing in making decisions relative to best-case uses of RTT and service time. The best performance is also dependent on the performance metric used. If better performance is obtained via one of these best-case approaches on a consistent basis then that result points that the current DNS mechanism for placing clients of these Webbased services could be improved.

In comparing the performance of these approaches for selecting a server relative to a particular performance metric we choose not to show performance directly, but rather show the difference of between the performance of an approach and the best performance observed for that metric within a group of requests. That is, for each group of requests, 
and for each metric, we find out the server with the best performance. We identify the server providing the best performance for each of the four metrics denoted as $S_{\text {best-firstbyte }}$, $S_{\text {best-total-pers }}, S_{\text {best-firstbyte, }}$ and $S_{\text {best-firstbyte-pers. In }}$ showing the results we compute the time differences between the best server for a metric and the servers identified by each of the three approaches. For example, $T_{\text {firstbyte }}\left(S_{d n s}\right)-$ $T_{\text {firstbyte }}\left(S_{\text {best-firstbyte }}\right)$ shows the difference of the first byte time between the server $S_{d n s}$ and the best server

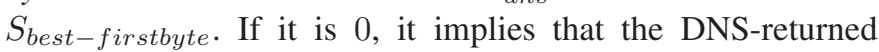
server is indeed the best server $\left(S_{d n s} \equiv S_{\text {best-firstbyte }}\right)$ in terms of this performance metric. Using a baseline allows us to focus on the relative differences between the performance of various servers because situations where all servers perform well or all perform poorly for a client are not server selection problems.

\section{Base Results for U.S. Client Set}

We ran a script on each client every 10 minutes where it invoked each of the 16 Web-based services (by retrieving the given URL) from each of the IP addresses known to the respective global- and U.S.-based client sets. We also did a DNS lookup of the corresponding server name for each service to know which IP address would be selected by the DNS mechanism at the time. These lookups and retrievals were made using a modified Perl LWP library to not only be able to send requests to the DNS returned IP but also to send requests to all IP addresses in our known set. Each group of requests was repeated 100 times over a 16-hour period. We dropped requests that took more than 10 seconds, which occurred for $0.03 \%$ of retrievals for the U.S. clients (and 6.5\%, primarily from $\mathrm{KR}$ and $\mathrm{AU}$, for the global clients). We repeated this methodology multiple times over a one-month period for our two client sets with the results shown from last round of data gathering. In this section we focus on the base results from the U.S. client set as a starting point.

Analysis of the results obtained from the experiments of the 16 services validated our expectation that the four Image services all demonstrated negligible services times and the DNS-identified server was often the same as the server with the best RTT. For the 'CNBC Search' service we observe only one server IP in our test so there is no notion of "selection". The 'FoxNews Search' service both yielded large service times for the first server accessed by a client as well as what appeared to be back-end caching effects that resulted in inconsistent results. As a result we dropped this service as well as the other Search and four Image services and concentrated our analysis on the remaining ten services.

Figure 2 shows the base performance results comparing DNS performance with best RTT and best service time for all tests for all clients of the ten remaining services using the first byte performance metric. As described in Section III-C, a difference of zero indicates that the given approach yields the best performance time for a client from amongst the set of all possible servers and shows that all approaches attain this result in close to $50 \%$ of the tests.

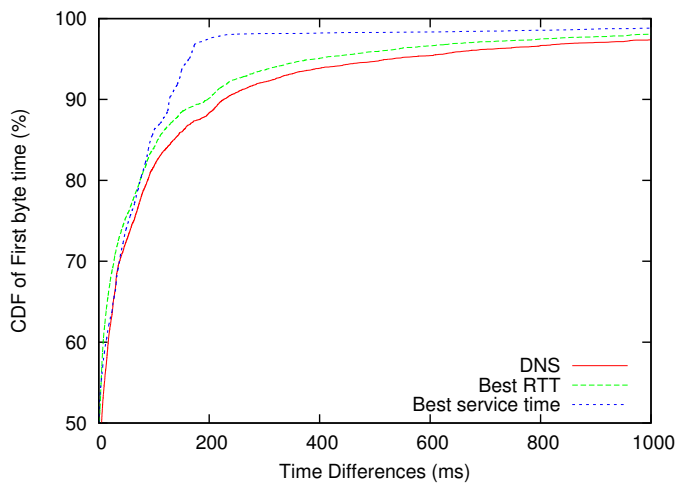

Fig. 2. Base Performance for All Services for All U.S. Clients

In general, most of them are doing reasonable jobs at most of the time using the DNS returned server. However, at the $85 \%$-tile there begins to be a noticeable performance difference between the DNS-returned server and the best possible server for this metric. The server providing the best RTT closely tracks the DNS-returned server performance, but about the $85 \%$-tile the servers providing the best service time yield noticeable better first byte time performance. This difference does not necessarily mean that a selection policy that takes into account service time will yield better decision as this is a best-case situation, but it does indicate that consideration of such an approach is warranted.

\section{Consideration of Service Time in Server SELECTION}

In Section IV we found that clients for some services are not being directed to their best servers, that is the DNS decision making could be improved. We also found that in some, but not all, cases consideration of the service time could improve the decision making. The question is how to implement a policy that can consider the service time without ignoring the RTT between client and server, which is still an important component in the decision. Rather than use a single approach for combining these two values, we define a simple parameterized function $T(N)$, which accounts for $T_{r t t}$ and $T_{\text {service }}$ with an integer parameter $N \geq 0$, i.e., $T(N)=T_{\text {service }}+N \times T_{r t t}$. As written this function assumes the service time and RTT are already known. Instead we define $\operatorname{Exp} T(N)$ as the next expected $T(N)$ using an exponential weighted average to update it over time, i.e.,

$$
\operatorname{Exp} T(N)=\alpha \times \operatorname{Exp} T(N)+(1-\alpha) \times T(N)
$$

Note that we tested different values of $\alpha$ and found similar results hence we report only those of $\alpha=0.7$ in this paper.

Finally, we define the policy $P_{N}$ as the one that always chooses the server with the minimum expected $T(N)$, i.e., $P_{N}=$ ChooseMin $(\operatorname{Exp} T(N))$. Intuitively, policy $P_{N=0}$ always chooses the server with the lowest 'Expected Service Time', while $P_{N=\infty}$ selects the server with the lowest 'Expected RTT'. In general, a policy with a smaller $N$ favors 'Service Time' over 'RTT', while a policy with a larger $N$ 
favors servers with a lower 'RTT'. In our work we specifically examine policies for $N=0,1,2$ and $\infty$.

\section{Simulation of Policies}

We evaluated the use of varying combinations of expected RTT and service time for different Web-based services using the collected data via trace-driven simulation. With 100 retrievals for each service, we used the first 10 retrievals to initially compute expected RTT and service times and then used the remaining 90 retrievals to both evaluate each policy as well as continue to update the expected value.

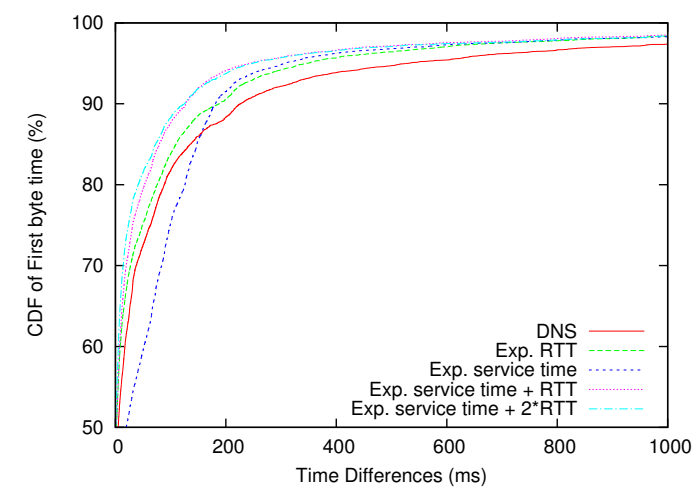

Fig. 3. Simulation of Policies for All Services for All U.S. Clients

Figure 3 shows the simulation results for the chosen 10 services. The results show that consideration of only the expected service time $\left(P_{N=0}\right)$ results in the worst performance over a range of the CDF while use of only the expected RTT $\left(P_{N=\infty}\right)$ performs comparable or a bit better than the DNS decision. However the $P_{N=1}$ and $P_{N=2}$ policies consistently perform as well or better than all of the other policies. This result is particularly encouraging as it occurs with a simple policy on a deployed production platform.

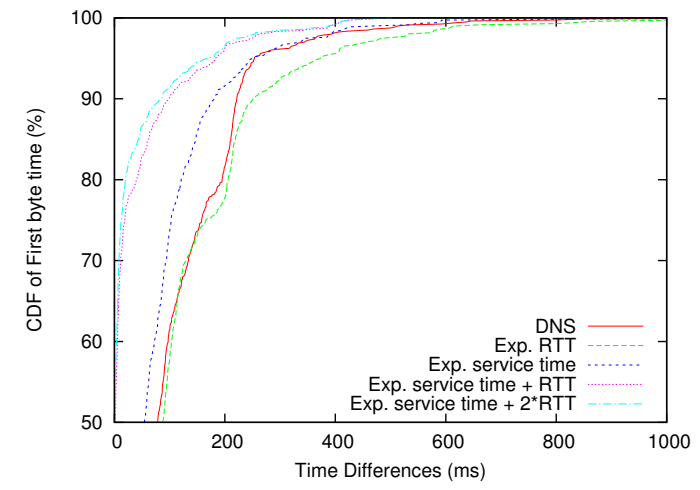

Fig. 4. Simulation of Policies for Fox News Home Page Service for All U.S. Clients

Figure 4 shows simulation results for the 'FoxNews Home Page' where a combination of expected RTT and service time (with $N=1$ or $N=2$ ) provides the best overall results. Figure 5 provides another view of simulation results for the Fox News Home Page Service by showing the 90\%-tile results on a per-client basis where the clients are shown in a west to east order. These results (more per-service results are in [2]) show that there is variation in performance amongst the policies even for different client locations of the same service, although the $P_{N=2}$ generally performs the best or close to the best for each client.

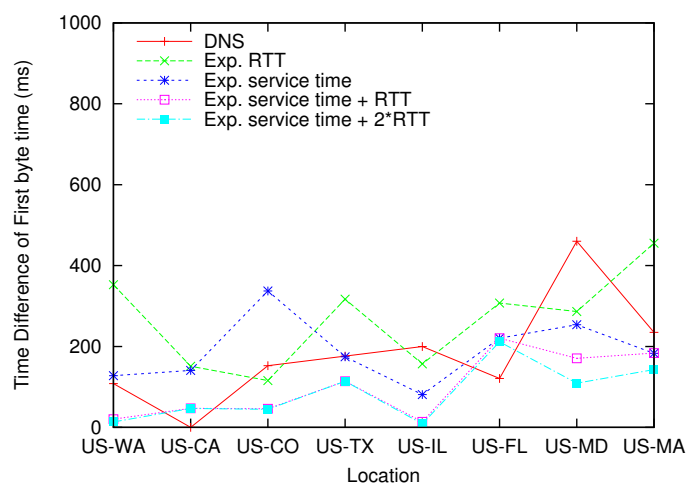

Fig. 5. Simulation of Policies for FoxNews Home Page Service for Specific U.S. Clients (90\%-tile First Byte Time Results)

Figure 6 shows results comparable to those shown in Figure 3 except the performance metric has changed from First Byte Time to Total Time. The total time reflects retrieval of all data for a service thus the effect of a larger RTT could delay data reception due to the TCP ACK mechanism. Thus it is not surprising that consideration of only the expected service time is not a good policy for this metric, but as shown in Figure 6 the other policies are competitive with DNS. The $P_{n=2}$ and $P_{n=\infty}$ policies even provide slightly better performance than DNS-selected server.

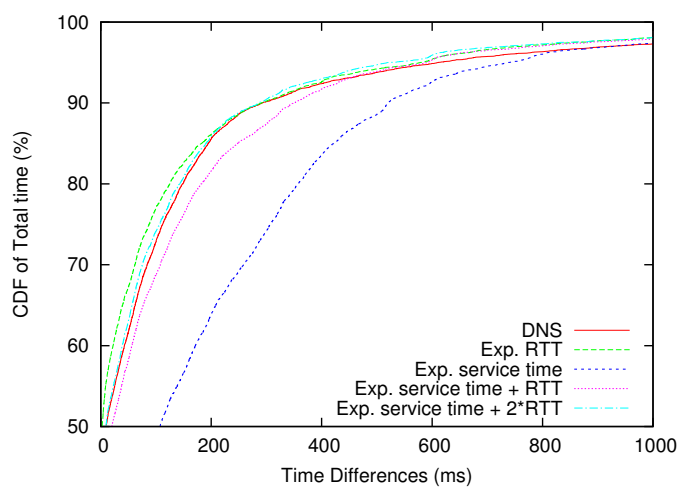

Fig. 6. Simulation of Policies for All Services for All U.S. Clients (Total Time)

As indicated in Section III, we performed a similar study for a set of global clients. Figure 7 shows the first byte time metric results for all 10 services for all of the 10 global clients. This figure is the same as Figure 3 except for a different client set. The results shown in Figure 7 are similar to those shown in Figure 3 except there is a clear performance penalty for only considering expected service time in the decision. Otherwise the $P_{N=1}$ and $P_{N=2}$ policies are comparable, if not better, results than obtained via DNS and expected RTT policies. The close performance between DNS and expected RTT indicates 
that RTT is the dominant component in current DNS decision making.

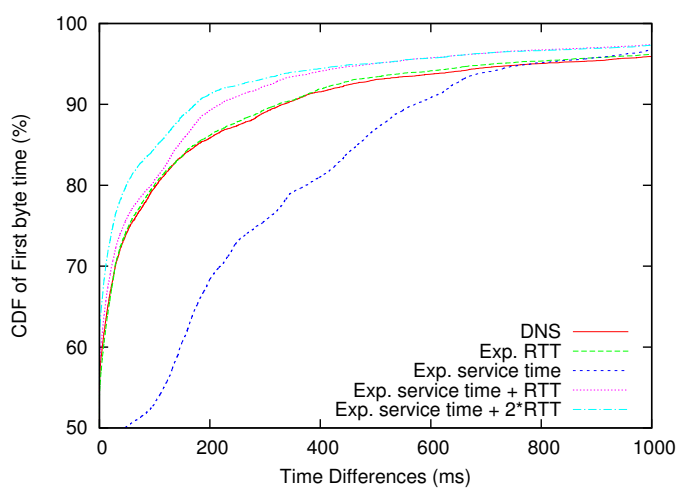

Fig. 7. Simulation of Policies for All Services for All Global Clients

\section{RELATED WORK}

Generally, in large-scale data centers, clients are redirected to different servers for the reasons of (i) improved client latency and (ii) providing load balancing. This is mostly done by using DNS to return server IPs of the closest data center to the clients. The effectiveness of this approach is studied in [3], [4]. However, one of the limitations for this approach is the need for name exposure. Using the same domain name for different services would result in making the same decision for clients requesting different services. Another limitation for prior work is the round-trip time and front-end server load are usually the dominant factors to consider when making decisions. Our work suggests Web sites should expose their service names in domain names for more refined decisions and proposes the consideration of service time in mapping clients to servers, which implicitly relates to the back-end server load.

Content Distribution Networks are a popular means to serve static content for web sites. Work in [5] had preliminary measurements on the performance of CDN content delivery. Google studied the performance of its own CDN and found out the causes for some large latencies appearing in their CDN [6]. Our work has a different point of view. We observe the performance from a client perspective and propose a method to account for significant back-end server response time.

In addition to mapping clients to appropriate data centers, resource provisioning is another direction for improving the Web performance. While not directly related, our work was partially inspired by the work of dynamic resource provisioning between multiple dependent services [7], [8]. Intuitively, allocating more resource to the back-end server may improve the 'Service Time', while putting more front-end servers closer to the clients may reduce the RTT. Although our approach does not reallocate any resource, we focus on how to make better use of current allocation. Our work may, in return, help others work on resource provisioning decisions.

\section{SUMmARY AND FUTURE WORK}

In this work we have explored the explicit use of backend service time for Web-based services in mapping clients of these services to specific client-facing servers that provide them. We gathered data from currently deployed server platforms of popular Web sites and used the decisions of the existing DNS mechanism as a benchmark for mapping clients to specific front-end servers. Using two distinct set of clientsone set located in the U.S. and another set located around the world-we first performed a best-case analysis to see if the front-end server providing the best service time (or best RTT) to the client would have given better performance than the server selected via DNS. We then went on to propose simple policies for the combination of expected RTT and service time. Evaluating these policies for a range of performance metrics with a trace-driven simulation over the collected data, we observed better performance by our policies compared with DNS for some services and comparable performance with DNS for the remaining services. Given the increasing use of Web-based services, these results are important in showing that client-perceived performance can be improved by consideration of back-end service time costs.

Moving forward, there is much opportunity for future work. We plan to extend our methodology to study the potential improvement of other currently deployed Web-based services to understand the applicability of our techniques. We also plan to examine the reasons that service times for a particular service are large when it is provided by some front-end servers, but not for others. There are multiple explanations such as a poorly performing front-end server, an over-loaded local backend server or the need to retrieve data from a remote backend server. There is also need to study this problem not only from the client perspective, but also to set up controlled server platforms so that we can measure performance on a platform with a known configuration for how Web-based services are provided.

\section{REFERENCES}

[1] "Alexa: Most popular web sites," http://www.alexa.com/.

[2] W. Zhang and C. E. Wills, "Consideration of Service Time in Placing Clients of Web-Based Services," Worcester Polytechnic Institute, Technical Report WPI-CS-TR-11-02, February 2011.

[3] J. Pang, A. Akella, A. Shaikh, B. Krishnamurthy, and S. Seshan, "On the responsiveness of dns-based network control," in Proceedings of the 4th ACM SIGCOMM conference on Internet measurement. New York, NY, USA: ACM, 2004, pp. 21-26.

[4] A. Shaikh, R. Tewari, and M. Agrawal, "On the effectiveness of DNSbased server selection," in Proceedings of the IEEE Infocom 2001 Conference, Anchorage, Alaska USA, April 2001.

[5] B. Krishnamurthy, C. Wills, and Y. Zhang, "On the use and performance of content distribution networks," in Proceedings of the ACM SIGCOMM Internet Measurement Workshop, San Francisco, November 2001, pp. 169-182.

[6] R. Krishnan, H. Madhyastha, S. Srinivasan, S. Jain, A. Krishnamurthy, T. Anderson, and J. Gao, "Moving beyond end-to-end path information to optimize CDN performance," in Proceedings of the 9th ACM SIGCOMM conference on Internet measurement conference. ACM, 2009, pp. 190201.

[7] D. Jiang, G. Pierre, and C. Chi, "Autonomous resource provisioning for multi-service web applications," in Proceedings of the 19th international conference on World Wide Web. ACM, 2010, pp. 471-480.

[8] B. Urgaonkar, P. Shenoy, A. Chandra, P. Goyal, and T. Wood, "Agile dynamic provisioning of multi-tier internet applications," ACM Trans. Auton. Adapt. Syst., vol. 3, no. 1, pp. 1-39, 2008. 